\title{
Version 5.0 de SwissDRG: des améliorations progressives
}

\author{
Petra Ingenpass ${ }^{a}$, Beatrix Meyer ${ }^{b}$ \\ ${ }^{a}$ Dr med., cheffe suppléante de la division Tarifs et économie de la santé pour les médecins hospitaliers \\ ${ }^{b}$ Cheffe de la division Tarifs et économie de la santé pour les médecins hospitaliers
}

\begin{abstract}
Dans la version 5.0 de SwissDRG, SwissDRG SA a procédé à des modifications concernant notamment la pédiatrie et la médecine palliative, différents cas complexes et coûteux, et les hospitalisations longues (high-outlier). En revanche, aucune solution n'a été trouvée pour plusieurs problèmes fondamentaux tels que le défaut de qualité des données et la rémunération souvent inappropriée des cas très déficitaires.
\end{abstract}

\section{Changements positifs pour la pédiatrie et les hospitalisations longues}

Dans la version 5.0 de SwissDRG, tous les DRG ont fait l'objet d'une analyse systématique concernant d'éventuels splits de cas en pédiatrie, débouchant sur la création de 47 nouvelles répartitions selon l'âge. A partir de 2016, le traitement très coûteux des nouveau-nés de moins de $750 \mathrm{~g}$ disposera de son propre DRG offrant une meilleure représentativité des coûts et marquant un retour par rapport à la condensation de DRG de la version 2.0 pour les cas inférieurs à $1000 \mathrm{~g}$ à la naissance. Par ailleurs, les enfants qui doivent suivre un traitement complexe atteignent le DRG correspondant avec moins de points de dépense que les adultes grâce à un rabaissement de la valeur-seuil. Selon les chiffres de SwissDRG SA, l'index de case-mix de la pédiatrie s'améliore de 3,68\% par rapport à la version précédente.

La représentativité des séjours supérieurs à la durée normale définie (high-outlier) a de nouveau fait l'objet d'une analyse pour la version 5.0 de SwissDRG. Le coût des médecins, les coûts des médicaments et du matériel médical sont désormais affectés aux coûts différentiels. De plus, SwissDRG SA a évalué chaque DRG séparément en fonction de différents critères occasionnant une hausse du supplément high-outlier pour 259 DRG.

\section{Davantage de rétributions additionnelles}

Pour la version 5.0 de SwissDRG, il a été possible de calculer 57 rétributions additionnelles [1], soit huit de plus que dans la version précédente, qui ont toutes été tarifées, sauf celles pour les systèmes d'assistance cardiaque ventriculaire gauche et droite. Trois ont été supprimées pour les médicaments car SwissDRG SA n'a pas relevé d'amélioration de la qualité tarifaire par le calcul de ces substances.

Il est réjouissant de voir que le nombre d'hôpitaux qui ont pris part aux relevés détaillés des données pour les médicaments, implants et procédures coûteux a sensiblement augmenté. Cependant, les hôpitaux n'ont pas toujours communiqué le prix d'achat des médicaments, si bien que, pour les rétributions additionnelles concer-

Plusieurs changements utiles sont réalisés sur une base de données encore insuffisante.

nées, SwissDRG a appliqué une réduction de 30\% sur le prix de fabrication comme il l'avait annoncé. Cette réduction pourrait même atteindre $40 \%$ dans la version 6.0 si les prix d'achat ne sont pas signalés. Là, une communication étroite entre SwissDRG SA et les hôpitaux est nécessaire afin d'éviter de telles réductions à l'avenir. Pour la FMH, il est par ailleurs primordial que les autres rétributions additionnelles déposées soient examinées en vue des versions à venir.

\section{Pas de solution en vue pour les cas très coûteux}

SwissDRG SA a analysé et actualisé différents cas de traitement coûteux et complexes, dont notamment la reconstruction plastique du sein en incluant pour la première fois dans le calcul des chiffres livrés en cours d'année comme la FMH l'avait déjà suggéré dans le passé. Mais la représentativité des cas très coûteux, et aussi très déficitaires, n'a connu aucune amélioration fondamentale alors que la stratégie 2013+ de SwissDRG SA la 
mentionnait déjà comme un développement prioritaire. En effet, les cas très coûteux déficitaires constituent une des raisons pour lesquelles le degré de couverture moyen des hôpitaux universitaires s'élève à seulement 90,7\% selon le modèle de calcul de SwissDRG SA avec un prix de base uniforme virtuel.

\section{SwissDRG n'est pas obligatoire pour la rémunération des soins palliatifs}

Une nouvelle pré-MDC (Major Diagnostic Category) a été créée pour permettre une rémunération plus différenciée et mieux adaptée aux dépenses des cas de traitement de soins palliatifs complexes, faisant ainsi disparaître, en contrepartie, les rétributions additionnelles

\section{Améliorer la qualité des données concernant} les investissements et les frais d'infrastructure par un soutien externe.

utilisées jusqu'à présent. Il s'agit maintenant de consolider l'expérience acquise avec cette nouvelle formule. Grâce à une proposition commune au conseil d'administration de SwissDRG SA et à un courrier de palliative.ch, la FMH et H+ ont obtenu la levée de l'obligation initialement prévue de facturer les soins palliatifs selon SwissDRG. Il appartient désormais aux partenaires tarifaires de savoir s'ils veulent négocier une rémunération selon SwissDRG ou s'ils préfèrent des forfaits journaliers.

\section{Importance de la coordination avec la révision de la CHOP}

Plusieurs changements et actualisations ont été mis en œuvre par SwissDRG SA concernant la logique du groupeur notamment dans le domaine de la chirurgie rachidienne alors que l'Office fédéral de la statistique (OFS) a procédé dans le même temps à des révisions ciblées de la classification des procédures $\mathrm{CHOP}$, accomplies avec le soutien des sociétés de discipline et de la FMH, en concertation avec SwissDRG SA. Il serait cependant pertinent de coordonner les procédures relatives aux changements dans les DRG avec les révisions de la CHOP: ainsi par exemple, la mise à jour de la CHOP dans le domaine de la chirurgie rachidienne pourrait être harmonisée avec les changements complexes du groupeur. Les propositions émises par la FMH sur le réajustement des tableaux des codes de procédures de la MDC 01 dans la version 0.4 suite à l'extension de la CHOP 2011 sont encore en attente d'être examinées par SwissDRG SA.

FMH Frohburgstrasse 15

CH-4600 Olten

Tél. 0313591111

Fax 0313591112

tarife.spital[at]fmh.ch

\section{Qualité des données: nette amélioration nécessaire}

D'après les documents mis à disposition par SwissDRG SA, la qualité des données sur les coûts et sur les prestations peut encore être nettement améliorée. Un contrôle plus important de la plausibilité des données livrées par les hôpitaux en 2013 s'est traduit par l'annulation des données de 22 hôpitaux, au lieu de six pour la version 4.0. Cela a concerné aussi bien le codage des prestations fournies que l'affectation des coûts aux cas particuliers. Si les efforts consentis jusqu'à présent et l'amélioration récente des règles pour la révision du codage sont à saluer, des mesures supplémentaires sont en revanche nécessaires.

La qualité des données concernant les investissements et les frais d'infrastructure (CUI) est toujours insuffisante. Comme l'année précédente, plus d'un tiers des hôpitaux n'ont pas présenté leurs données selon REKOLE $^{\circledR}$. Mais il faut noter que la part moyenne des investissements et frais d'infrastructure oscille de $2 \%$ à $19 \%$ des frais d'exploitation suivant les hôpitaux, une variation encore très importante qui était de $2 \%$ à $17 \%$ dans la version 4.0. Aucune méthode spécifique pour contrôler la plausibilité des investissements et des frais d'infrastructure n'a été transmise à SwissDRG SA.

\section{Nécessité d'aller plus loin}

Plusieurs des changements et des adaptations apportés à la version 5.0 de SwissDRG l'ont été sur une base de données de qualité insuffisante. Ici, des mesures supplémentaires pour améliorer les données sur les coûts et sur les prestations des hôpitaux sont nécessaires. Par ailleurs, la FMH recommande à SwissDRG SA d'attribuer un mandat externe en vue d'établir un concept pour une qualité améliorée des données, et notamment de proposer des méthodes de contrôle de la plausibilité des investissements et des frais d'infrastructure. SwissDRG SA a procédé à de nombreux changements dans la version 5.0 de la structure tarifaire qui entrera en vigueur à partir de 2016. Plusieurs des domaines concernés ont aussi fait simultanément l'objet d'importantes mises à jour par l'OFS dans la CHOP. C'est pourquoi il serait souhaitable que SwissDRG SA établisse une feuille de route en concertation avec l'OFS dans le but de définir les priorités du développement à moyen terme.

1 Micafungine, sunitinibe, erlotinibe, eculizumab, ustekinumab immunoglobuline anti-lymphocytes Thumains, panitumumab tarification différenciée de différentes aphérèses thérapeutiques, différenciation pour l'implantation sélective de spirales métalliques (coils) en localisation extra-/intracrânienne resp. intraspinale resp. périphérique, formes d'application différenciées: abatacept intraveineux et sous-cutané, trastuzumab intraveineux et sous-cutané, rituximab intraveineux et sous-cutané. 